\title{
Visualization of Contact Interaction of Nanobeams
}

\author{
M.V. Zhigalov ${ }^{1}$, V.A. Apryskin ${ }^{1}$, V.A. Krysko ${ }^{1}$ \\ zhigalovm@yandex.ru|wwooow@yandex.ru \\ ${ }^{1}$ Yuri Gagarin Saratov State Technical University, Saratov, Russia
}

The paper presents a visualization of the contact interaction of two Bernoulli-Euler nanobeams connected through boundary conditions. Mathematical models of beams are based on the gradient deformation theory and the theory of contact interaction of B. Y. Cantor. The visualization is based on Fourier transform and wavelet transform, phase portrait.

Keywords: Bernoulli-Euler nanobeam, gradient deformation theory, contact problem, Fourier transform, wavelet.

\section{Introduction}

The designs of modern devices are complex multi-layer packages with small gaps between the elements, so an important issue is to take into account the contact interaction of layers, which in turn leads to a strong nonlinearity constructive. Such nano electro-mechanical systems (NEMS) are widely used in various electronic devices, in particular in gyroscopes (layered flat micromechanical accelerometers (MMA). Note that the account of contact interaction leads to the chaotic state of the system already at small amplitudes of oscillations. The presence of a gap between the elements, such as beams, already at small deflections, commensurate with the gap between the elements, can lead the object under study in a state of chaotic oscillations. Therefore, the need for research with visualization of the contact interaction of nano-mechanical systems in the form of beams is undoubtedly relevant and requires attention.

\section{Statement of the problem}

Micro-and nano-sized beams are widely used in various micro-and nano-Electromechanical systems (vibration sensors [1], micro-drives [2], micro-switches [3]). The dependence of elastic behavior on the body size at the micron scale was observed experimentally in different substances (metals [4, 5] and alloys [6], polymers [7], crystals [8]).

The dependences of elastic behavior on size can be explained using molecular dynamics (MD) simulations or higher order continuum mechanics. Although the molecular dynamics approach can provide more accurate approximations to real objects, it is too expensive from a computational point of view. Therefore, the higher order continuum mechanics approach has been widely used in the modeling of small-scale structures.

The development of a higher order continuum theory can be traced back to the earliest work of Piola in the 19th century, as shown in [9], and the work of the Kosser brothers [10] in 1909. However, the ideas of the Kosser brothers received considerable attention from researchers only since the 1960s, when a large number of higher-order continuum theories were developed. In general, these theories can be divided into three different classes, namely, the family of strain gradient theories, the microcontinuum, and nonlocal elasticity theories.

Based on the higher order stress theory of Mindlin [11] and Lam et al. [12] proposed the theory of elasticity of the deformation gradient, in which, in addition to the classical equations of equilibrium of forces and moments, a new additional equilibrium equation is introduced, which determines the behavior of stresses of higher orders and the equation of equilibrium of moments. Three parameters of the material length scale are introduced for isotropic linear elastic materials $\left(l_{0}, l_{1}, l_{2}\right)$. According to this theory, the total strain energy density is a function of the symmetric strain tensor, the dilation gradient vector, the deviator tension gradient tensor, and the symmetric rotation gradient tensor.

In this paper, a mathematical model of Bernoulli-Euler nanobeams connected through boundary conditions under the action of transverse load is constructed. Three material length scale parameters are introduced to account for dimensional effects $\left(l_{0}, l_{1}, l_{2}\right)$. To account for the contact between the beams, a Winkler coupling between the compression and the contact pressure between the two beams is used [13]:

$$
\Psi=\frac{1}{2}\left[1+\operatorname{sign}\left(w_{1}-h_{k}-w_{2}\right)\right],
$$

where $\Psi=1$, if $\mathrm{w}_{1}>w_{2}+h_{k}$ To that is, there is contact between the plate and the beam, else $\Psi=0, \mathrm{w}_{1}, w_{2}, h_{k}$ deflections of the first and second beams and the gap between them, respectively.

The mathematical model of contact between two nanobeams, based on the kinematic Bernoulli-Euler hypothesis, is described by a system of resolving equations:

$$
\begin{gathered}
\frac{\partial^{4} w_{m}}{\partial x^{4}}\left((\lambda+2 \mu) \frac{b h^{3}}{12}+l_{0}^{2} \mu b h+l_{2}^{2} \mu b h+\frac{96}{225} l_{1}^{2} \mu b h\right)- \\
-\frac{\partial^{6} w_{m}}{\partial x^{6}}\left(2 l_{0}^{2} \mu \frac{b h^{3}}{12}+\frac{14}{225} l_{1}^{2} \mu \frac{b h^{3}}{12}\right)-q_{m}+ \\
+(-1)^{m} K\left(w_{1}-w_{2}-\tilde{h}\right) \Psi=\frac{\partial^{2} w}{\partial t^{2}}+\varepsilon \frac{\partial w}{\partial t},
\end{gathered}
$$

where $m$ - beam number $(m=1,2), \tilde{h}$ - the gap between the beams.

Boundary conditions are:

$$
w_{m}=0 ; \frac{\partial w_{m}}{\partial x}=0 .
$$

Initial conditions are:

$$
w_{m}(x, 0)=0 ; \frac{\partial w_{m}(x, 0)}{\partial t}=0 .
$$

The system (2-4) was reduced to the Cauchy problem using the finite difference method $\mathrm{O}\left(h^{2}\right)$. The Cauchy problem was solved by the Runge-Kutta method of 4 orders. A study of convergence by the method of finite differences, on the basis of which the optimal number of partitions was chosen, was carried out. The partitioning step for the Runge-Kutta method was determined according to the Runge principle.

\section{The results of the study of the influence of length scale factors on the nature of oscillations}

For the considered tasks the following parameter values were used: $a / h=30, q_{1}=10 \sin (5.3 \cdot t), q_{2}=0, \varepsilon=0.1$, $\tilde{h}=0.01$.

The research results for two of the nine considered combinations of coefficients $\left(l_{0}, l_{1}, l_{2}\right)$ are shown in Figure 1, 2. For the first case (Fig. 1), all coefficients are zero, i.e. considered beams on the classical theory. The second case (Fig. 
2) is characterized by the values $l_{0}=0.3 ; l_{l}=0.3 ; l_{2}=0.3$, i.e. all three dimensional factors are taken into account. The figures in the first row show the deflections of the first $w_{1}$ and second beams $w_{2}$. The second line shows the phase synchronization of oscillations $\varphi_{1}-\varphi_{2}$, marked in dark color. The third and fourth lines show the Fourier spectrum $S(\omega)$, the wavelet spectrum based on the Morlet maternal wavelet, and the phase portrait $w(\dot{w})$ for the first and second beams, respectively. Phase synchronization is based on the approach described in [14-15]. When analyzing phase chaotic synchronization, it is necessary to enter the phase $\phi(t)$ of chaotic signals [16, 17]. Frequencies of chaotic signals are defined as average rates of change of the phases of signals $\langle\dot{\phi}(t)\rangle$. Wavelet analysis can be applied to identify the phase synchronization mode of mechanical dynamic systems with a poorly defined phase. The behavior of such systems is characterized on the basis of continuous sets of phases, which are determined using continuous wavelet transform of a chaotic signal $w(t)[16,17]$. If the beams in question are not in the phase synchronization mode, their behavior is asynchronous on all time scales. As soon as any of the time scales of the considered dynamic systems are synchronized, the phase synchronization mode arises. Obviously, the time scales that have the maximum power of the wavelet spectrum are synchronized first of all. The remaining time scales are still unsynchronized. Therefore, phase synchronization leads to the occurrence of phase capture on synchronized time scales.

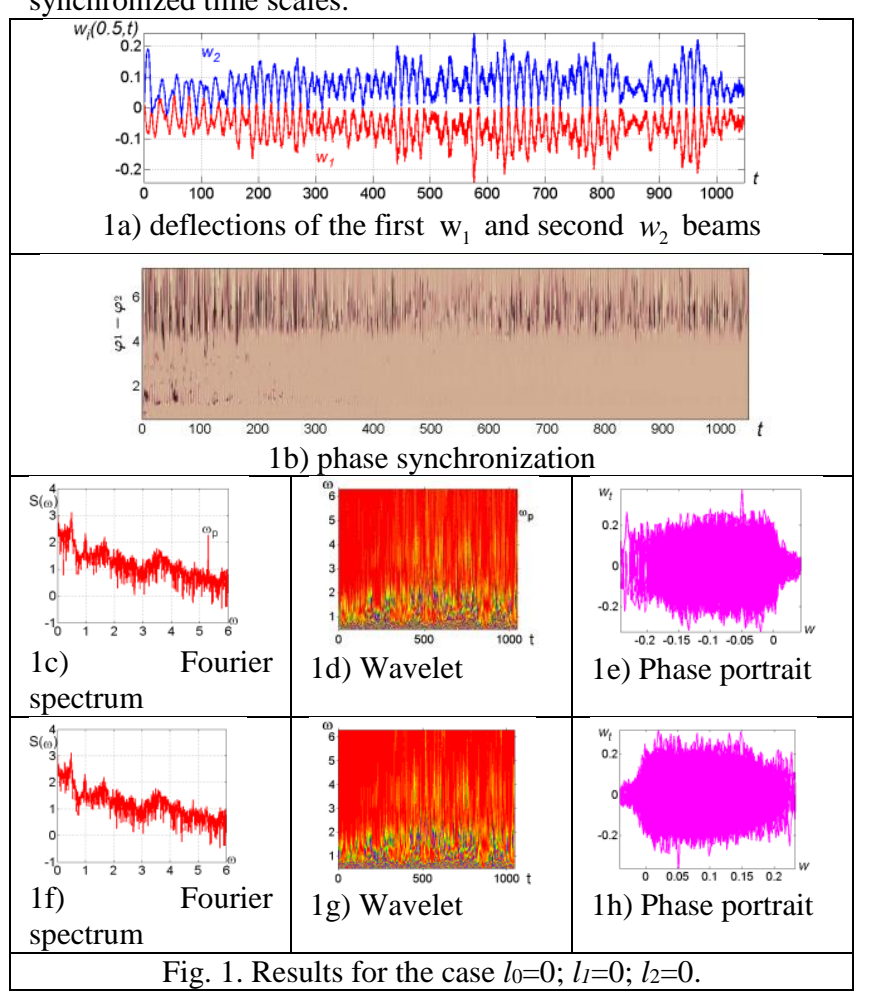

Graphs deflection changes in time is shown on the complex vibrations. And before the $t \approx 160$ deflections change synchronously. This reflects the phase synchronization graph in the second row of the table. There are two synchronization zones on this graph: at the excitation frequency $\omega_{p}=5.3$ and at

the lower frequency $\omega \approx 2$. In the rest of the time interval, the oscillations of the two beams are practically "mirrored", and synchronization occurs only at the excitation frequency $\omega_{p}=5.3$.
The Fourier spectrum for both beams indicates a chaotic state of the system, while both spectra are qualitatively similar and have several pronounced frequencies in the low range $\omega_{i} \approx 3.5 ; 1.5$. Wavelet spectra indicate that these frequencies appear in the spectrum with $t \approx 200$. Moreover, the frequency spectrum varies significantly over time. Phase portraits of beam oscillations are "mirrored".
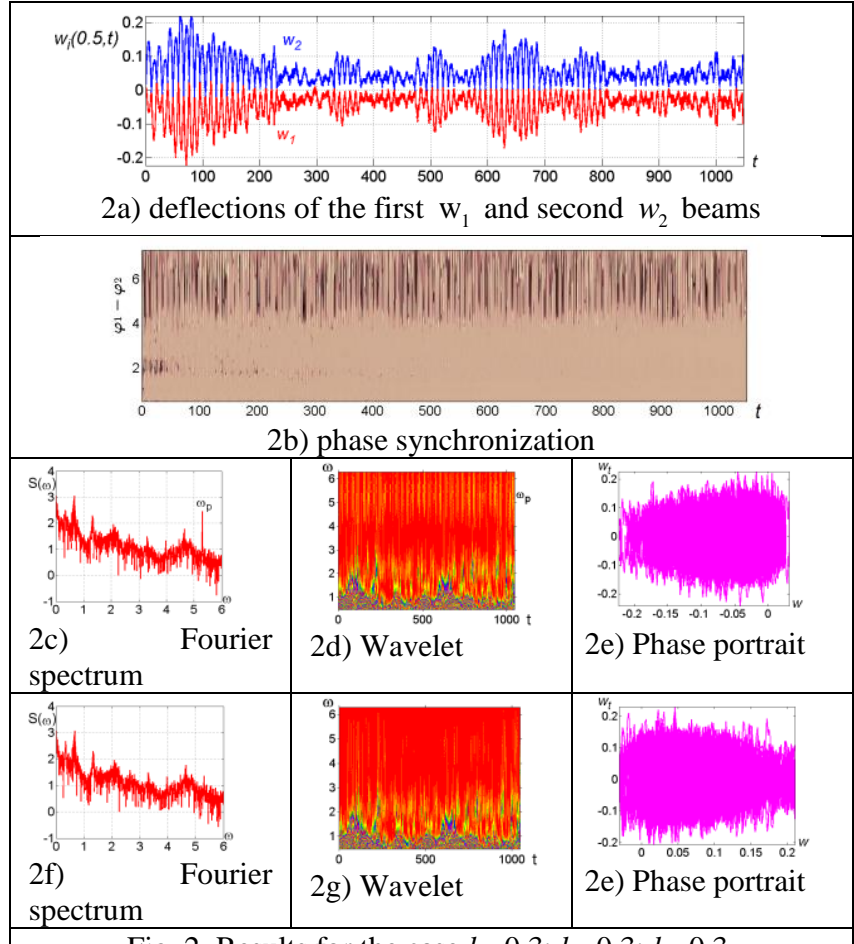

Fig. 2. Results for the case $l_{0}=0.3 ; l_{l}=0.3 ; l_{2}=0.3$.

Consider the case when both beams are described according to the theory of the strain gradient taking into account the values of $l_{0}=0.3 ; l_{1}=0.3 ; l_{2}=0.3$. Graphs of deflections (signals) differ significantly from the previous case. The synchronism of oscillations is present at a longer interval up to $t \approx 200$. The phase synchronization graph shows that in this time interval synchronization occurs at two frequencies: at the excitation frequency $\omega_{p}=5.3$ and at the frequency $\omega \approx 2$, as in the previous case. However, synchronization at the excitation frequency is uniform over the entire time interval, in contrast to the previous case, where phase synchronization is practically absent in the interval $t \in(480 ; 600)$. The Fourier spectrum for both beams is qualitatively similar, as in the previous case, but there are differences. The number of frequencies with the highest energy (peaks in the spectrum) is much larger and their distribution differs significantly from the previous case. Thus, the contact interaction energy of two nanobeams differs significantly from the beams according to the classical theory, and therefore, when studying the contact of NEMS elements, it is necessary to use the theory of size-dependent behavior. The wavelet spectrum also has a significant difference from the previous case. For the first beam, both the excitation frequency and all frequencies of the range are present throughout the time interval $\omega \in(0 ; 3)$. The frequencies of the other bands appear at the initial time $t \in(0 ; 230)$ and at the end of the time interval for contact interaction studies. The phase portraits of both beams, as well as for the previous case, are "mirrored", but qualitatively different. They also differ quantitatively by the values of the velocity of oscillations $\dot{w}$. 


\section{Conclusion}

In the work, on the basis of the built-in mathematical model of the Bernoulli-Euler nanobeam, the influence of taking into account the size-dependent coefficients on the character of the contact interaction is studied. The necessity of taking into account the theory of higher order for the study of NEMS elements in the form of beams is shown. The visualization of contact interaction characters based on the wavelet transform, both for studying phase chaotic synchronization and for studying changes in the oscillation spectrum over time, allows us to more fully consider various aspects of contact interaction.

\section{Appreciation}

This work was supported by the Russian Science Foundation, grant № 16-11-10138-П.

\section{References}

[1] Fu, Y., Zhang, J. Electromechanical dynamic buckling phenomenon in symmetric electric fields actuated microbeams considering material damping. Acta Mech. 212, (2010), 29-42. [2] M. Moghimi Zand, and M. T. Ahmadian, "Static pull-in analysis of electrostatically actuated microbeams using homotopy perturbation method", Appl. Math. Model. 34 (2010), 1032-1041.

[3] Jia, X.L., Yang, J., Kitipornchai, S.: Pull-in instability of geometrically nonlinear micro-switches under electrostatic and Casimir forces. Acta Mech. 218, (2011), 161-174.

[4] Fleck, N.A., Muller, G.M., Ashby, M.F., Hutchinson, J.W., 1994. Strain gradient plasticity: theory and experiments. Acta Metall. Mater. 42, 475-487.

[5] Nix, W.D. Mechanical properties of thin films. Metall. Trans. A 20, (1989), 2217-2245.

[6] E. Mazza, S. Abel,J. Dual, Experimental determination of mechanical properties of Ni and Ni-Fe microbars, Microsystem Technologies 2 (4) (1996), 197-202

[7] Lam DCC, Yang F, Chong ACM, Wang J, Tong P Experiments and theory in strain gradient elasticity. J Mech Phys Solids 51: (2003) 1477-1508

[8] Q. Ma,D. R. Clarke, Size dependent hardness of silver single crystals, Journal of Materials Research 10 (4) (1995) 853-863.

[9] Dell'Isola F, Della Corte A, Esposito R, Russo L. Some cases of unrecognized transmission of scientific knowledge: From antiquity to Gabrio Piola's peridynamics and generalized continuum theories. Generalized Continua as Models for Classical and Advanced Materials: Springer; (2016). p. 77-128. [10] Cosserat E, Cosserat F. Théorie des corps déformables: Paris: Hermann et Fils, 1909.

[11] Mindlin RD. Second gradient of strain and surface-tension in linear elasticity. Int J Solids Struct. (1965);1:417-38.

[12] Yang F, Chong ACM, Lam DCC, Tong P. Couple stress based strain gradient theory for elasticity. Int J Solids Struct. (2002) ;39:2731-43.

[13] Kantor B. Ya/, Bogatyrenko T. L. A method for solving contact problems in the nonlinear theory of shells. Dokl. Ukrain Academy of Sciences. Ser A 1; (1986). pp 18-21.

[14] Krysko V.A., Koch M.I., Zhigalov M.V., Krysko A.V. "Chaotic Phase Synchronization of Vibrations of Multilayer Beam Structures" Journal of Applied Mechanics and Technical Physics. 2012. T. 53. № 3. C. 451-459.

[15] J. Awrejcewicz, M.V. Zhigalov, V.A. Krysko-jr., U. Nackenhorst, I.V. Papkova, A.V. Krysko, 'Nonlinear dynamics and chaotic synchronization of contact interactions of multilayer beams', in: 'Dynamical Systems - Theory', Eds. J. Awrejcewicz, M. Kaźmierczak, P. Olejnik, J. Mrozowski, TU of Lodz Press, 2013, 283-292 (ISBN 978-83-7283-588-8)
[16] Osipov G.V., Pikovsky A.S., Rosenblum M.G., Kurths J. Phys.rev. Lett. 1997. V. 55. P.2353.

[17] Pikovsky A.S., Rosenblum M.G., Kurths J. Synhronization: a Universal Cocept in Nonlinear Sciences. Cambridge University Press, 2001. 